\title{
Chemokine expression profile of freshly isolated human glioblastoma-associated macrophages/microglia
}

\author{
KIRSTEN HATTERMANN ${ }^{1}$, SUSANNE SEBENS ${ }^{2}$, OLE HELM ${ }^{2}$, ANNE DOROTHÉE SCHMITT ${ }^{3}$, \\ ROLF MENTLEIN $^{1}$, H. MAXIMILIAN MEHDORN ${ }^{3}$ and JANKA HELD-FEINDT ${ }^{3}$ \\ ${ }^{1}$ Department of Anatomy, University of Kiel, D-24098 Kiel; ${ }^{2}$ Institute for Experimental Medicine, \\ Inflammatory Carcinogenesis and ${ }^{3}$ Department of Neurosurgery, University Medical Center \\ Schleswig-Holstein UKSH, Campus Kiel, D-24105 Kiel, Germany
}

Received February 25, 2014; Accepted April 2, 2014

DOI: $10.3892 /$ or.2014.3214

\begin{abstract}
Several studies have substantiated the hypothesis that tumor progression is not only driven by the tumor cells themselves but also by their interaction with intrinsic and surrounding stromal cells. Tumor-associated macrophages and microglial cells (TAMs) represent one major stromal cell component of glioblastomas. Additionally, in many gliomas, chemokines are highly expressed and some chemokines were already linked to settlement of TAMs in tumors. However, although chemoattraction mechanisms mediated by chemokines and their receptors are well documented, information on their expression and role in TAMs, particularly in patients, is limited. Therefore, we investigated the transcription of the chemokine-receptor combinations CXCL12-CXCR4-CXCR7, CXCL16-CXCR6 and CX3CL1-CX3CR1 in freshly isolated TAMs from 20 human glioblastomas in relation to in vitro polarized M1- and M2-macrophages. We demonstrated that TAMs express both M1- and M2-markers. Compared to in vitro polarized macrophages, the M1-marker interleukin (IL)-6 was similarly expressed, whereas IL-1 $\beta$ and tumor necrosis factor (TNF)- $\alpha$ were found at lower levels. The M2-marker IL-10 was comparably expressed, while CD163 and transforming growth factor (TGF)- $\beta$ were detected with one tenth lower intensities in TAMs. All investigated chemokines/receptors were transcribed at moderate to high levels in TAMs as well as in vitro polarized macrophages. However, CX3CR1 was markedly higher and CXCR7 was somewhat higher expressed in TAMs, whereas M2-macrophages were characterized by the highest
\end{abstract}

Correspondence to: Professor Janka Held-Feindt, Department of Neurosurgery, University Medical Center Schleswig-Holstein, UKSH, Campus Kiel, Arnold-Heller-Str. 3, Building 41, D-24105 Kiel, Germany

E-mail: held-feindtj@nch.uni-kiel.de

Abbreviations: GAPDH, glyceraldehyde-3-phosphate dehydrogenase; GBM, glioblastoma; GFAP, glial fibrillary acidic protein; TAM, tumor-associated macrophages and microglia; Iba-1, ionized calcium-binding adapter molecule

Key words: chemokine, glioma, microglia, macrophages
CXCL12 and a moderate CX3CL1 expression. Collectively, TAMs share properties of M1- and M2-macrophages and show a considerably higher expression of the chemokine receptors CXCR7 and CX3CR1.

\section{Introduction}

For many years, cancer was predominantly considered to be a cell-autonomous disease. However, studies on the tumor microenvironment have given rise to the emerging concept that tumor progression depends on a dense network of interactions among cancer cells and the surrounding stroma, including different types of immune cells (1). For glioblastomas (GBMs), high malignant brain tumors with a median survival of $<15$ months despite multimodal therapy (2) tumor-associated macrophages and microglia (TAMs) are the predominant infiltrating immune cell population. This population is characterized by $\mathrm{CD}_{11} \mathrm{~b}^{+} / \mathrm{CD} 45^{\mathrm{dim}}$ (microglia) and $\mathrm{CD} 11 \mathrm{~b}^{+} / \mathrm{CD} 45^{\text {high }}$ (macrophages) phenotypes and accounts for $13-34 \%$ (microglia) and $4.2-12 \%$ (macrophages) of the tumor cell mass in experimental gliomas (3). There is compelling evidence that TAMs are involved in creating a microenvironment that favors glioma growth (4-6). They are regarded as M2-macrophages, which exhibit anti-inflammatory properties as they show elevated expression of the mannose (CD204/206) and scavenger receptor (CD163) as well as an enhanced expression and secretion of immunosuppressive cytokines, such as transforming growth factor (TGF)- $\beta 1$ and IL-10 (7). In contrast, the classically activated or M1-polarized macrophages are characterized by a pro-inflammatory phenotype, including enhanced expression and release of IL-1 $\beta$, tumor necrosis factor (TNF)- $\alpha$, interleukin (IL)- 6 or IL-12 along with the ability to induce Th1-mediated immune responses (7). However, the precise molecular mechanisms underlying the phenomena of tumorprogression facilitated by TAMs, remain unclear. Since it is known that TAMs could be recruited to sites of gliomas by an interplay of CCL2/MCP-1 (constitutively produced by glioma cells) and its receptor CCR2 (expressed on TAMs) $(8,9)$ and by a CCL7/MCP-3- CCR1/CCR2/CCR3- crosstalk (10), it seems that small chemotactic cytokines, known as chemokines, are important key players in GBM progression by facilitating the infiltration of TAMs into glioma tissues. In accordance 
with this, we have previously shown that CX3CL1 promotes recruitment of TAMs into GBM and enhances expression of the matrix metalloproteases (MMPs) 2, 9 and 14 in these tumor cells (11). To date, there are no data available regarding the expression of the transmembrane chemokine CXCL16 and its receptor CXCR6 and regarding the expression of CXCL12 with both its receptors CXCR4 and CXCR7 on TAMs freshly isolated from GBM. Since we and others showed that both CXCL16 and CXCL12 and their respective receptors are involved in GBM progression (12-17), the elucidation of these chemokine/-receptors on TAMs will help to unravel the role of TAMs in glioma and may promote the design of more effective treatment options for these tumors.

Therefore, the present study demonstrated the expression profile of CX3CL1/CX3CR1, CXCL16/CXCR6 and CXCL12/CXCR4/CXCR7 in both freshly isolated human GBM-associated macrophages/microglia and in in vitro generated M1- and M2-macrophages and related these results to the M1- and M2-marker expression profile of the different macrophage populations.

\section{Materials and methods}

CD11b MACS separation procedure for TAM isolation. Twenty different freshly obtained human GBM samples were used for CD11b MACS separation technology as previously described (11). Therefore, single-cell suspensions of $400 \mathrm{mg}$ tumor tissue were generated using the Neural Dissociation Kit (T), and up to $2 \times 10^{7}$ cells were immediately labeled with CD11b MicroBeads (Miltenyi Biotec GmbH, Bergisch Gladbach, Germany) and separated using MACS LS columns according to the manufacturer's instructions. Then, the identity of freshly isolated CD11b positive TAMs was confirmed by immunofluorescence using a monoclonal mouse anti-human CD11b FITC-labeled antibody (Miltenyi Biotec $\mathrm{GmbH}$ ) according to the manufacturer's instructions (10 $\mu \mathrm{l}$ undiluted CD11b-FITC for up to $10^{7}$ separated cells). Additionally, mRNA expression levels of glial fibrillary acidic protein (GFAP; glioma-cell specific) and ionized calcium-binding adapter molecule (Iba-1; monocyte/microglia-specific) of isolated human TAM-enriched fractions and non-TAM fractions were evaluated by qPCR (as described below). Only TAM preparations which were CD11b positive compared to corresponding non-TAM fractions and exhibited high Iba-1 mRNA expression were used for further analysis. Materials were obtained in accordance with the Helsinki Declaration of 1975 and with approval of the Ethics Committee of the University of Kiel.

Generation of M1- and M2-macrophages. Macrophages were generated from monocytes from healthy adult blood donors obtained from the Research Center Borstel, Germany. Informed consent was obtained from all donors. Monocytes ( $15 \times 10^{6} ; \sim 95 \%$ purity) generated from human PBMCs by counterflow centrifugation (elutriation) (18) were cultured in teflon-coated bags (Süd-Laborbedarf, Gauting, Germany) in RPMI-1640 medium containing 1\% L-glutamine (both from PAA Laboratories, Cölbe, Germany), 10\% FCS and $100 \mu \mathrm{g} / \mathrm{ml}$ penicillin/streptomycin (both from Biochrom, Berlin, Germany) in the presence of either $50 \mathrm{ng} / \mathrm{ml} \mathrm{M-CSF}$ or GM-CSF (BioLegend, Fell, Germany) for 7 days. To detach macrophages, bags were placed on ice for $1 \mathrm{~h}$ and cells were resuspended in PBS (PAA Laboratories). The phenotype of macrophages was characterized following established protocols $(19,20)$ and as recently described $(21)$.

Quantitative real-time $R T-P C R$ ( $q P C R)$. Twenty different TAM-enriched and non-TAM fractions as well as five individual in vitro generated M1- and M2-macrophage preparations were used. Total RNA from these cells $\left(>1 \times 10^{3}\right)$ was purified with the PicoPure RNA Isolation kit (MDS Analytical Technologies, Sunnyvale, CA, USA) according to the manufacturer's instructions. The RNA samples were treated with RNase-free DNase (1 U/ $\mu$ l) (Promega, Madison, WI, USA), and reverse transcribed by RevertAid ${ }^{\mathrm{TM}}$ H Minus M-MuLV Reverse Transcriptase (200 U/ $\mu \mathrm{l})$ (Fermentas, Vilnius, Lithuania) as previously described $(11,15)$. Quantitative PCR was performed in triplicate using a total reaction volume of $20 \mu \mathrm{l}$, containing $1 \mu \mathrm{l}$ of 20X Assays-on-Demand ${ }^{\mathrm{TM}}$ Gene Expression Assay Mix (Iba-1, Hs_00610419_g1; GFAP, Hs_00157674_m1; CX3CL1, Hs_00171086_m1; CX3CR1, Hs_00365842_m1; CXCL12, Hs_00171022_m1; CXCR4, Hs_00237052_m1; CXCR7, Hs_00664172_m1; CXCL16, Hs_00222859_m1; CXCR6,Hs_00174843_m1; CD163, Hs_00195682_m1; IL-10, Hs_00961619_m1; TGF- $\beta 1$, Hs_00171257_m1; TNFa, Hs_00174128_m1; IL-1 $\beta$, Hs_01555410_m1 and IL-6, Hs_00985639_m1; Applied Biosystems, Foster City, CA, USA), $10 \mu \mathrm{l}$ of 2X TaqMan Universal PCR Master Mix and 100 or 10 ng of cDNA template (diluted in RNase-free water to $9 \mu \mathrm{l})$. After $2 \mathrm{~min}$ at $50^{\circ} \mathrm{C}$ and $10 \mathrm{~min}$ at $95^{\circ} \mathrm{C}, 40$ cycles of $15 \mathrm{sec}$ at $95^{\circ} \mathrm{C}$ and $1 \mathrm{~min}$ at $60^{\circ} \mathrm{C}$ were performed. Glyceraldehyde3-phosphate dehydrogenase (GAPDH, Hs99999905_m1; Applied Biosystems) mRNA was amplified in each sample as internal positive control. Each plate included at least three 'no template controls (NTC)'. The reaction was carried out with the MyiQ ${ }^{\mathrm{TM}}$ Single-Color Real-time PCR Detection System (Bio-Rad, Munich, Germany) and fluorescence data were converted into $\mathrm{C}_{\mathrm{T}}$ measurements. $\Delta \mathrm{C}_{\mathrm{T}}$ values of each sample were calculated as: $\mathrm{CT}_{\text {gene of interest }}-\mathrm{CT}_{\mathrm{GAPDH}}$. Relative gene expression was calculated with $2^{\text {(normalized } \mathrm{CT} \text { non-stimulated - normalized }}$ CT stimulated) $=n$-fold of control. $\Delta \mathrm{C}_{\mathrm{T}}=3.33$ corresponds to one order of magnitude. Low $\Delta \mathrm{C}_{\mathrm{T}}$ values indicate high expression.

\section{Results}

TAM enrichment and characterization. To obtain further insight into the expression of M1- and M2-marker as well as chemokines and their receptors in human TAMs, we isolated this cell type from 20 different fresh solid human GBM samples using well-established CD11b MACS separation technology (11). As fresh human GBM samples were mostly of small size (max. $400 \mathrm{mg}$ tumor weight), CD11b separation technology had to be performed in micro format and analytical methods were essentially limited to qPCR.

Fig. 1 shows qPCR results of enriched human TAM and non-TAM fractions measured for expression of the microglial/macrophage-specific molecule Iba-1. Iba-1 mRNA expression differed only slightly between individual TAM-enriched fractions (mean $\Delta \mathrm{C}_{\mathrm{T}}$ value, 3.40), and was clearly detected at higher levels in TAM-enriched vs. non-TAM fractions (mean $\Delta C_{T}$ value, 9.60). Since a $\Delta C_{T}$ 


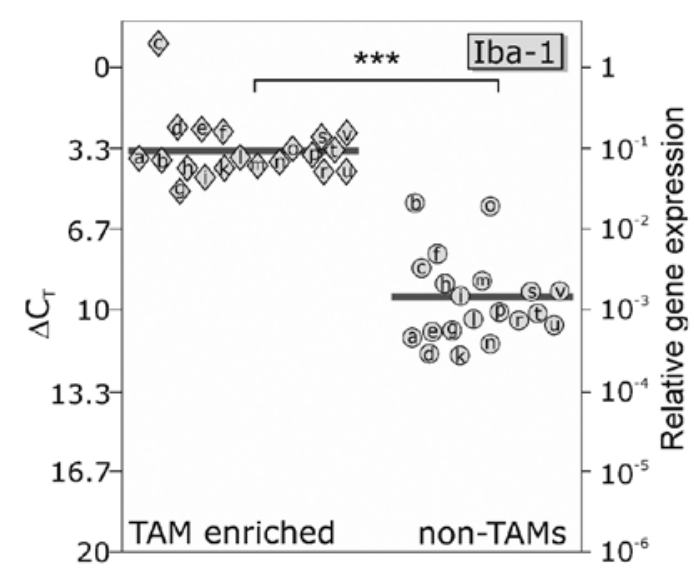

Figure 1. Enrichment of tumor-associated macrophages/microglia (TAMs) from 20 fresh human glioblastoma samples. TAMs were enriched by MACS separation, and Iba-1 transcription was determined by quantitative RT-PCR. Iba-1 was significantly higher transcribed in TAM-enriched fractions compared to the remaining non-TAM fractions (mean, 75-fold higher), with mean $\Delta \mathrm{C}_{\mathrm{T}}$ values of 3.4 (TAM-enriched) and 9.63 (non-TAMs). Iba-1, ionized calcium-binding adapter molecule.

difference of 3.33 corresponds to one order of magnitude, for Iba-1 a 75.0-fold higher expression in TAM-enriched vs. non-TAM fractions was measured $(\mathrm{p}<0.001)$, indicating an accurate isolation procedure.

We investigated the M1- and M2-marker expression profile of freshly isolated TAM-enriched fractions and observed that both M1- and M2-markers were detectable in TAMs but with different expression intensities (Fig. 2). Regarding M1-specific markers, IL-1 $\beta$ and TNF $\alpha$ were found at clearly lower levels in TAM-enriched fractions in relation to in vitro polarized M1-macrophages, whereas IL-6 mRNA was expressed in approximately similar amounts (Fig. 2, top). In detail, mean $\Delta \mathrm{C}_{\mathrm{T}}$ value for IL-1 $\beta$ was 0.056 in TAMs (vs. -2.09 in M1-polarized macrophages), mean $\Delta \mathrm{C}_{\mathrm{T}}$ value for TNF $\alpha$ was 6.44 in TAMs (vs. 2.11 in M1-polarized macrophages), and mean $\Delta \mathrm{C}_{\mathrm{T}}$ value for IL-6 was 4.78 in TAMs (vs. 4.14 in M1-polarized macrophages). Thus, the mean TAM-enriched fractions were characterized by a 4- and 20-fold lower expression of IL- $1 \beta$ and TNF $\alpha$, respectively, but exhibited somewhat similar IL-6 expression amounts compared to M1-polarized macrophages.

For M2-specific markers, CD163 and TGF $\beta$ were transcribed in lower amounts in TAM-enriched fractions in comparison to in vitro M2-polarized macrophages, whereas IL-10 showed somewhat similar transcription amounts in TAM-enriched fractions and M2-polarized macrophages (Fig. 2, bottom). In detail, mean $\Delta \mathrm{C}_{\mathrm{T}}$ value for $\mathrm{CD} 163$ was 1.01 in TAMs (vs. -2.60 in M2-polarized macrophages), mean $\Delta \mathrm{C}_{\mathrm{T}}$ value for TGF $\beta$ was 3.08 in TAMs (vs. -0.52 in M2-polarized macrophages), and mean $\Delta \mathrm{C}_{\mathrm{T}}$ value for IL-10 was 5.64 in TAMs (vs. 5.09 in M2-polarized macrophages). Thus, TAMs were characterized by a 12-fold lower mean expression of both CD163 and TGF $\beta$, but nearly equal expression of IL-10 compared to M2-polarized macrophages.

Chemokine expression profile of TAM-enriched fractions. The expression patterns of the two transmembrane chemokines CXCL16 and CX3CL1 with their corresponding receptors

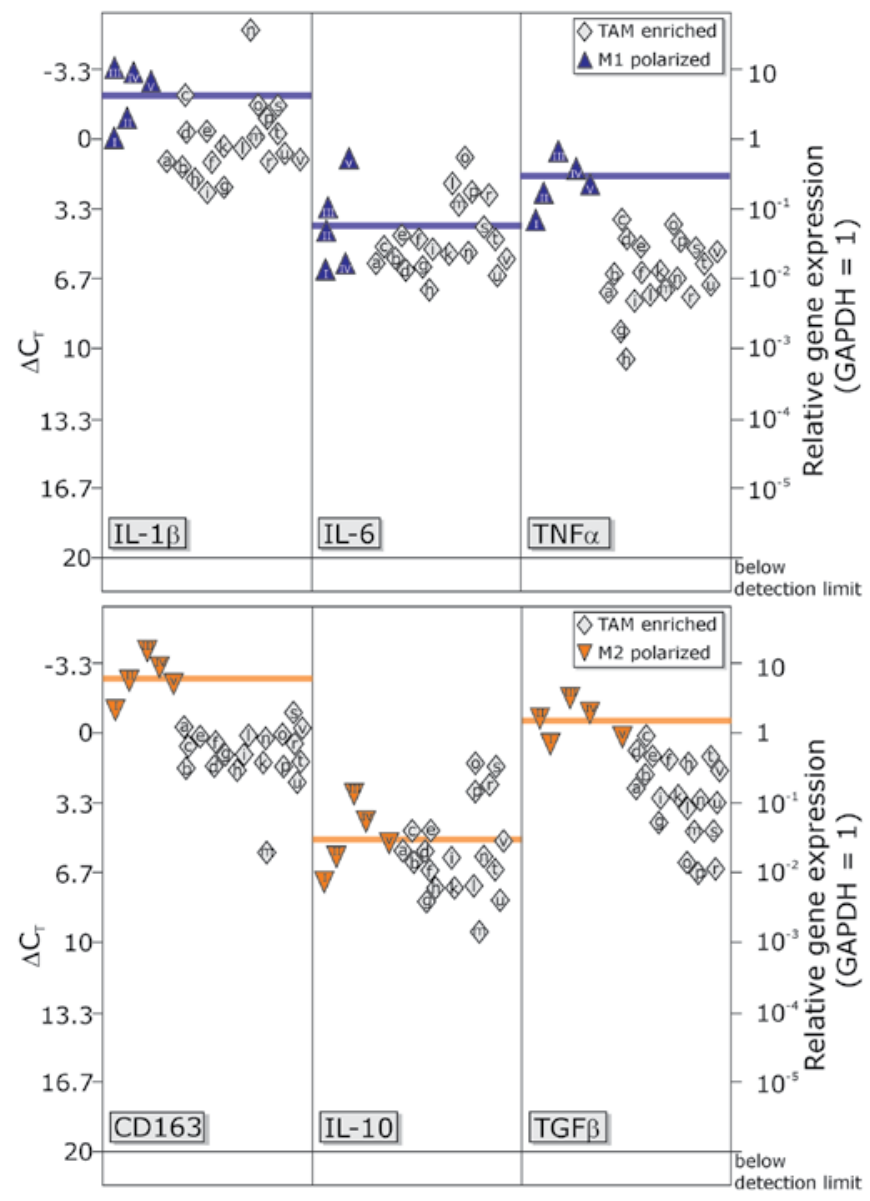

Figure 2. Transcription levels of M1- and M2-markers in TAM-enriched fractions. TAM-enriched fractions were analyzed by quantitative RT-PCR for transcription of M1- and M2-macrophage markers IL-1 $\beta$, IL-6 and TNF $\alpha$ (M1), and CD163, IL-10, TGF $\beta$ (M2). All markers were transcribed at well detectable levels. In comparison to in vitro M1-polarized macrophages (by differentiation with GM-CSF) from healthy human donors, mean transcription of IL-1 $\beta$ and TNF $\alpha$ was significantly lower in TAM-enriched samples, whereas IL-6 transcription was almost similar. Regarding in vitro M2-polarized macrophages (by differentiation with M-CSF), CD163 and TGF $\beta$ expression were significantly lower in TAM-enriched samples while almost equally high transcription patterns were observed for IL-10. TAM, tumor-associated macrophages and microglia.

CXCR6 and CX3CR1 as well as the expression of CXCL12 with both its known receptors CXCR4 and CXCR7 were determined in TAM-enriched fractions as well as M1- and M2-polarized macrophages (Fig. 3).

In general, all investigated chemokines and receptors were transcribed at moderate to high levels in TAM-enriched fractions (except for CXCR6) and M1-/M2-polarized macrophages (except for CX3CL1). Analysis of CXCL12 and its receptors CXCR4 and CXCR7 (left part of Fig. 3) revealed that CXCL12 transcription was $~ 48$-fold higher in M2-polarized macrophages compared to M1-polarized macrophages (mean $\Delta \mathrm{C}_{\mathrm{T}}$ value, 1.75 vs. 7.34 ), and the mean transcription level of TAM-enriched fractions ranged between these two pure macrophage populations (mean $\Delta \mathrm{C}_{\mathrm{T}}$ 5.58) with variations of the single preparations ranging from M2- to M1-macrophage levels. The receptors CXCR4 and CXCR7 were almost equally expressed in M1- and M2-macrophages [mean $\Delta \mathrm{C}_{\mathrm{T}}$ values, 4.19 and 2.68 (CXCR4), 5.86 and 6.31 (CXCR7)], and in TAM-enriched fractions CXCR4 was despite some outliers to 


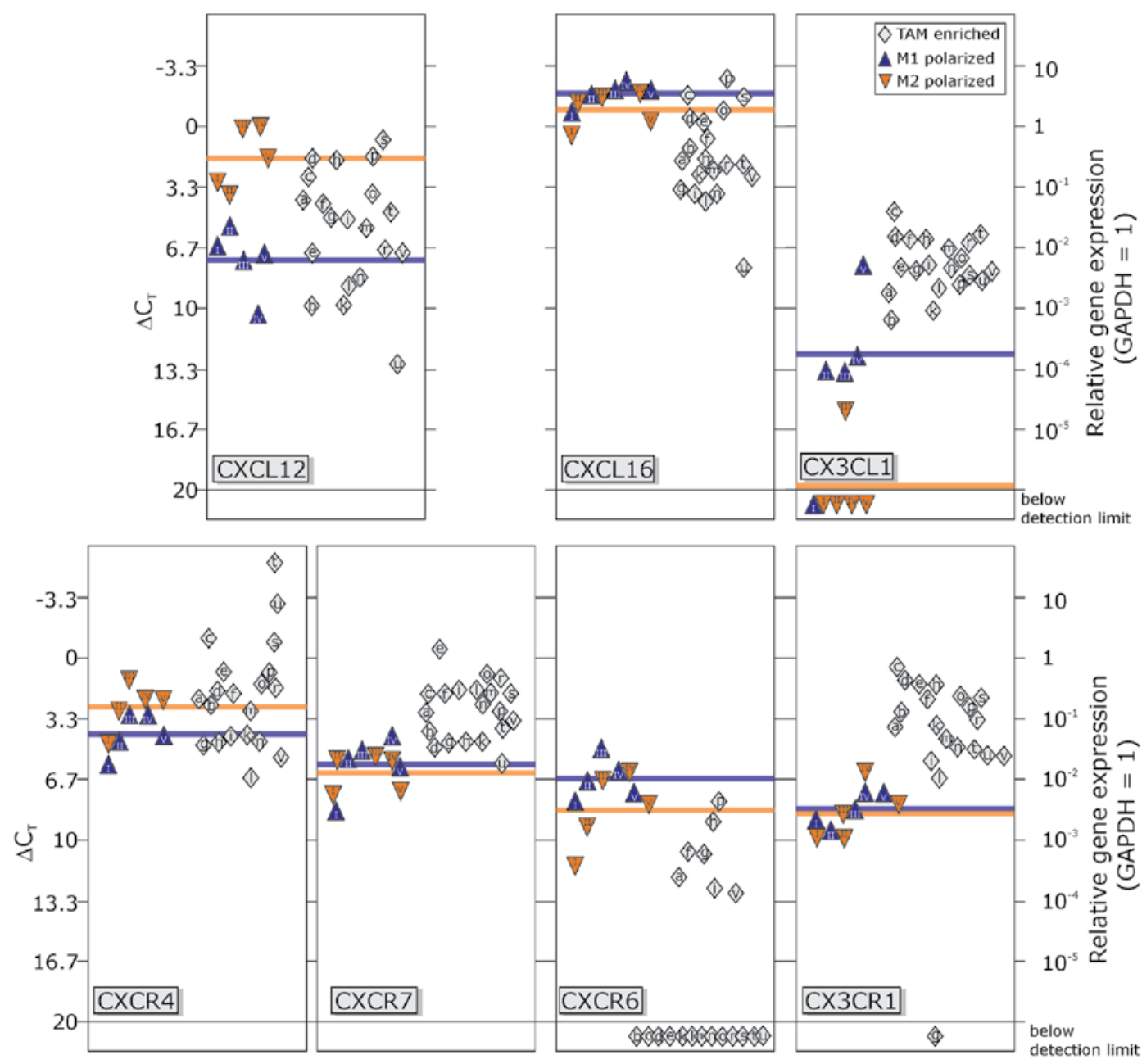

Figure 3. Transcription levels of chemokines and their receptors in TAM-enriched fractions in comparison to M1- or M2-polarized macrophages. The chemokine CXCL12 was expressed at moderate levels in M1-polarized macrophages, but was 10 -fold higher in M2-polarized macrophages. The transcription in TAM-enriched fractions showed considerable variation, between high mRNA levels comparable to M2-macrophages and moderate levels as in M1-macrophages. The expression of the two CXCL12 receptors, CXCR4 and CXCR7, was very similar in M1- and M2-polarized macrophages; in TAM-enriched samples, transcription of CXCR4 was comparable to both polarized macrophage subsets, but slightly elevated for CXCR7. In both in vitro polarized macrophages, expression of the chemokine CXCL16 was very high with only slight variations, also between M1- and M2-macrophages. In contrast, in enriched TAMs, CXCL16 expression was clearly lower, but still at high expression levels. The only known receptor for CXCL16, CXCR6 was expressed at moderate levels in polarized macrophages, but only rarely found in TAMs (7/20 samples). Expression of CX3CL1 (fractalkine) was low in M1-polarized macrophages, nearly absent in M2-polarized macrophages, but detectable in TAM-enriched fractions. In contrast, the fractalkine receptor CX3CR1 was moderately found in M1- and M2-polarized macrophages and even 10-fold higher in TAM-enriched cell fractions. TAM, tumor-associated macrophages and microglia.

higher values in this range (mean $\Delta \mathrm{C}_{\mathrm{T}}$ value, 2.03), whereas mean CXCR7 expression (mean $\Delta \mathrm{C}_{\mathrm{T}}$ value, 2.84 ) was $\sim 10$-fold higher compared to both M1- and M2-macrophages.

Regarding the expression of CXCL16 and CXCR6, M1- and M2-macrophages both exhibited a very high expression of CXCL16 (mean $\Delta \mathrm{C}_{\mathrm{T}}$ values, -1.82 and -0.90 ), while TMA-enriched fractions showed 5-10-fold lower levels (mean $\Delta \mathrm{C}_{\mathrm{T}}$ value, 1.64). Markedly, this expression amount was even higher than expression levels in total GBM samples previously reported by Hattermann et al (13). In contrast, the corresponding receptor CXCR6 was rarely detectable in TAM-enriched fractions although it was present in all M1- and $\mathrm{M} 2$-polarized macrophage preparations (mean $\Delta \mathrm{C}_{\mathrm{T}}$ values, 6.64 and 8.36).

Although expression of CX3CL1, the ligand of CX3CR1, was quite low in M1-macrophages (mean $\Delta \mathrm{C}_{\mathrm{T}}$ value, 11.80), and mostly absent in M2-macrophages, it was elevated ( 17 -fold compared to M1) in TAM-enriched fractions (mean $\Delta \mathrm{C}_{\mathrm{T}}$ value, 7.70) but not as prominent as expression of CXCL12 and CXCL16 (as glioma cells highly produce both chemokines, this could, however, be caused by a certain contamination). The receptor CX3CR1 was almost similarly expressed in M1- and M2-polarized macrophages (mean $\Delta \mathrm{C}_{\mathrm{T}}$ values, 9.19 and 8.74), and markedly higher (55- and 40-fold) in TAM-enriched fractions (mean $\Delta \mathrm{C}_{\mathrm{T}}$ value, 3.42).

In summary, as shown in Fig. 3, TAM-enriched fractions represent quite homogenous populations without considerable variations for the expression of CXCR7, CXCL16 and CXCR6, CX3CL1 and CX3CR1. In the case of CXCR7, CX3CL1 and CX3CR1, the mean expression levels were higher than those of M1- and M2-macrophages, while the expression of CXCL16 and CXCR6 was lower. For both CXCL12 and CXCR4, the transcription levels varied between expression amounts of M1- and M2-polarized macrophages 
(with some outliers for CXCR4 with very high expression levels) and displayed a very heterogeneous expression pattern with greater differences between single TAM-enriched fractions.

\section{Discussion}

Since tumor-associated macrophages and microglia (TAMs) create a microenvironment that favors glioma growth, an understanding of the molecular pathways and signaling molecules affecting gene expression which lead to the altered phenotype of glioma TAMs is of considerable importance. Thus, several investigations focused on the role of TAMs in gliomas and sought to elucidate exactly how the interplay between TAMs and tumor cells is regulated $(4,22,23)$. It becomes clear that pro-tumor functions of TAMs are derived from their ability e.g. to regulate angiogenic programming and to provide soluble factors to malignant cells favoring proliferation, survival and invasion (23). Nevertheless, these diverse activities depend on the polarization state of TAMs that seems to be, beside others, regulated by cytokines and chemokines (23).

Chemokines are a family of peptide mediators that play essential roles in cellular migration and intercellular communication and were originally discovered as inducible cytokines facilitating the recruitment of specific leukocyte subsets. However, it is now well accepted that their role is not limited to inflammatory reactions, but extends to various other physiological and pathophysiological responses $(24,25)$. Chemokine and chemokine receptors are upregulated in a variety of human cancers suggesting that they play multifaceted roles in malignancy (26-29) and TAMs invasion and gene expression pattern is known to be clearly regulated by different chemokine/-receptors $(11,30)$. Thus, here, we analyzed the expression of CXCL16, CXCR6, CX3CL1, CX3CR1, CXCL12, CXCR4 and CXCR7 in freshly isolated human GBM-associated macrophages/microglia and in in vitro generated M1- and M2-macrophages and related these results to the M1- and M2-marker expression profile of these different cell types.

Initially, we showed that freshly isolated TAMs of human GBMs were characterized by both M1- and M2-specific markers. In detail, in comparison to M1-polarized macrophages, TAMs showed reduced IL- $1 \beta$ and TNF- $\alpha$ expression, whereas IL-6 mRNA level was somewhat similar. Additionally, in relation to M2-polarized macrophages, CD163 and TGF $\beta$ were found in lower amounts, whereas IL-10 was expressed at the same levels. Therefore, it seems that GBM-associated macrophages/microglia share properties of both 'end-differentiation-types' of M1- and M2-polarized macrophages. These results are in accordance with previous findings demonstrating that TAMs summarize a number of functions rendered by M2-polarized macrophages such as tuning of inflammatory responses and adaptive angiogenesis, tissue remodeling and repair and promoting of angiogenesis (31). Nevertheless, it was reported that TAMs also express IFN-inducible chemokines (32). As described by the authors themselves, a tight distinction between M1- and M2-macrophages does not fully represent the continuum of functional states that macrophages can express and is rather a simplified view (31). In this context, TAMs of GBM tissues seem to exhibit more functions of M2-polarized macrophages concomitantly preserving properties of M1-characteristics as has recently been shown by us for TAMs from pancreatic cancer patients (33).

Compared to M1- and M2-polarized macrophages, GBM-associated macrophages/microglia were characterized by a unique chemokine-specific profile. In detail, for CXCR7, CXCL16 and CXCR6 expression amounts of these chemokines were below those of M1- and M2-polarized macrophages; for CX3CL1 and CX3CR1 expression amounts found in TAMs were higher than those of different polarized macrophages; and for CXCL12 and CXCR4 expression amounts corresponded to those observed for M1- and M2-polarized macrophages. Collectively, CXCL16, CXCL12, CXCR4 and CXCR7 seem to be highly expressed, whereas CX3CL1, CX3CR1 and CXCR6 were lower transcribed in all investigated macrophage populations.

Since chemokines play a pivotal role in tumor progression, not only in GBMs but also in many other tumor types, these results are notable. For example, it has been shown that TAMs could be recruited to tumor cells by the expression of CCL2/MCP-1, CCL57RANTES and CXCL1 as products of tumor or stromal cells (34-36). Especially for CCL2, the level of tumor-derived CCL2 correlates with amounts of TAMs in several types of adenocarcinoma (37,38). Additionally, it is well accepted that the chemokine ligand-receptor pair CXCL12/CXCR4 is implicated in neoangiogenesis $(31,39)$, and CXCR4 is selectively upregulated in TAMs tumor and stromal cells (40). Moreover, TAMs can release chemokines that preferentially attract $\mathrm{T}$ cell subsets devoid of cytotoxic functions by release of, among others, CCL18, CCL17 and CCL22 $(31,38,41)$. However, high levels of Th1 chemokines such as CXCL9 and CXCL10 are also expressed by TAMs which suggested deviation from the typical M2 features of TAMs $(42,43)$.

For GBMs, we and others have shown that the chemokine/receptor pairs CXCL12/CXCR4/CXCR7, CXCL16/CXCR6 and CX3CL1/CX3CR1 are clearly involved in tumor progression (11-15). Here, we showed that GBM-associated macrophages/microglia are also characterized by expression of these chemokine/-receptor pairs indicating a pivotal role of this expression profile in TAM biology in gliomas. This aspect becomes more important since a clear tumor-promoting role of TAMs in glioma progression has been demonstrated in a recent report by Pyonteck et al (44). Her group showed that the inhibition of the colony-stimulating factor-1 in a GBM model significantly increased survival and suppressed growth of established tumors. Moreover, under these conditions, expression of M2-markers decreased in TAMs, which is consistent with their impaired tumor-promoting functions. In line with these results, Pong et al (45) demonstrated that F11R expression, a microglia surface marker, correlates positively with glioma malignancy grade and negatively with patient survival independent of the GBM molecular subtype. Additionally, Ye et al (46) stated that tumor-associated microglia/macrophages enhance the invasion of glioma stem-like cells via the TGF- $\beta 1$ signaling pathway.

In summary, TAMs are important mediators in glioma progression and one aspect of this progression is the chemokine 
profile of these high malignant brain tumors. Here, we showed for the first time that freshly isolated human GBM-associated TAMs share properties of pro- and anti-inflammatory macrophages and are characterized by an abundant expression of the chemokines/-receptors CXCL12/CXCR4/CXCR7, CXCL16/ CXCR6 and CX3CL1/CX3CR1 underscoring the potential of these chemokines/-receptor pairs as possible therapeutic targets.

\section{Acknowledgements}

The authors thank B. Rehmke, F. Ebrahim and J. Krause for their technical assistance. This study was supported by grants from the University of Kiel, the 'Deutsche Forschungsgemeinschaft' (HE3400/5-1 and ME758/10-1) and the BMBF (The PopGen 2.0 Network (P2N); support code: 01EY1103).

\section{References}

1. Elinav E, Nowarski R, Thaiss CA, Hu B, Jin C and Flavell RA: Inflammation-induced cancer: crosstalk between tumours, immune cells and microorganisms. Nat Rev Cancer 13: 759-771, 2013.

2. Ohgaki $\mathrm{H}$ and Kleihues P: Epidemiology and ethiology of gliomas. Acta Neuropathol 109: 93-108, 2005.

3. Badie B and Schartner JM: Flow cytometric characterization of tumor-associated macrophages in experimental gliomas. Neurosurgery 46: 957-961, 2000.

4. Li W and Graber MB: The molecular profile of microglia under the influence of glioma. Neuro Oncol 14: 958-978, 2012.

5. Markovic DS, Glass R, Synowitz M, Rooijen Nv and Kettenmann H: Microglia stimulate the invasiveness of glioma cells by increasing the activity of metalloprotease- 2 . J Neuropathol Exp Neurol 64: 754-762, 2005.

6. Zhai H, Heppner FL and Tsirka SE: Microglia/macrophages promote glioma progression. Glia 59: 472-485, 2011.

7. Mantovani A, Germano G, Marchesi F, Locatelli M and Biswas SK: Cancer-promoting tumor-associated macrophages: new vistas and open questions. Eur J Immunol 41: 2522-2525, 2011.

8. Kielian T, van Rooijen N and Hickey WF: MCP-1 expression in CNS-1 astrocytoma cells: implications for macrophage infiltration into tumors in vivo. J Neurooncol 56: 1-12, 2002.

9. Galasso JM, Stegman LD, Blaivas M, Harrison JK, Ross BD and Silverstein FS: Experimental gliosarcoma induces chemokine receptor expression in rat brain. Exp Neurol 161: 85-95, 2000.

10. Okada M, Saio M, Kito Y, Ohe N, Yano H, Yoshimura S, Iwama $\mathrm{T}$ and Takami T: Tumor-associated macrophage/microglia infiltration in human gliomas is correlated with MCP-3, but not MCP-1. Int J Oncol 34: 1621-1627, 2009.

11. Held-Feindt J, Hattermann K, Müerköster SS, Wedderkopp H, Knerlich-Lukoschus F, Ungefroren H, Mehdorn HM and Mentlein R: CX3CR1 promotes recruitment of human gliomainfiltrating microglia/macrophages (GIMs). Exp Cell Res 316: 1553-1566, 2010.

12. Hattermann $\mathrm{K}$ and Mentlein R: An infernal trio: the chemokine CXCL12 and its receptors CXCR4 and CXCR7 in tumor biology. Ann Anat 195: 103-110, 2013.

13. Hattermann K, Held-Feindt J, Ludwig A and Mentlein R: The CXCL16-CXCR6 axis in glial tumors. J Neuroimmunol 260: 47-54, 2013.

14. Hattermann K1, Held-Feindt J, Lucius R, Müerköster SS, Penfold ME, Schall TJ and Mentlein R: The chemokine receptor CXCR7 is highly expressed in human glioma cells and mediates antiapoptotic effects. Cancer Res 70: 3299-3308, 2010.

15. Hattermann K, Ludwig A, Gieselmann V, Held-Feindt $J$ and Mentlein R: The chemokine CXCL16 induces migration and invasion of glial precursor cells via its receptor CXCR6. Mol Cell Neurosci 39: 133-141, 2008.

16. Domanska UM, Kruizinga RC, den Dunnen WF, TimmerBosscha H, de Vries EG and Walenkamp AM: The chemokine network, a newly discovered target in high grade gliomas. Crit Rev Oncol Hematol 79: 154-163, 2011.
17. Sciumè G, Santoni A and Bernardini G: Chemokines and glioma: invasion and more. J Neuroimmunol 224: 8-12, 2010.

18. Grage-Griebenow E, Zwatzky R, Kahlert H, Brade L, Flad H and Ernst E: Identification of a novel dendritic cell-like subset of $\mathrm{CD} 4^{+} / \mathrm{CD} 6^{+}$blood monocytes. Eur J Immunol 31: 48-56, 2001.

19. Krausgruber T, Blazek K, Smallie T, Alzabin S, Lockstone H, Sahgal N, Hussell T, Feldmann M and Udalova IA: IRF5 promotes inflammatory macrophages polarization and $\mathrm{T}_{\mathrm{H}} 1-\mathrm{T}_{\mathrm{H}} 17$ responses. Nat Immunol 12: 231-238, 2011.

20. Verreck FA1, de Boer T, Langenberg DM, van der Zanden L and Ottenhoff TH: Phenotypic and functional profiling of human proinflammatory type-1 and anti-inflammatory type- 2 macrophages in response to microbial antigens and IFN- $\gamma$ - and CD40L-mediated costimulation. J Leukoc Biol 79: 285-292, 2006.

21. Schäfer H, Struck B, Feldmann EM, Bergmann F, GrageGriebenow E, Geismann C, Ehlers S, Altevogt P and Sebens S: TGF- $\beta 1$-dependent L1CAM expression has an essential role in macrophage-induced apoptosis resistance and cell migration of human intestinal epithelial cells. Oncogene 32: 180-189, 2013.

22. Kennedy BC, Showers CR, Anderson DE, Anderson L, Canoll P, Bruce JN and Anderson RCE: Tumor-associated macrophages in glioma: friend or foe? J Oncol 2013: 486912, 2013.

23. Ruffel B, Affara NI and Coussens LM: Differential macrophage programming in the tumor microenvironment. Trends Immunol 33: 119-126, 2012.

24. Miller RJ, Rostene W, Apartis E, Banisadr G, Biber K, Milligan ED, White FA and Zhang J: Chemokine action in the nervous system. J Neurosci 28: 11792-11795, 2008.

25. Vandercappellen J, Van Damme J and Struyf S: The role of CXC chemokines and their receptors in cancer. Cancer Lett 267: $226-244,2008$

26. Kakinuma T and Hwang ST: Chemokines, chemokine receptors, and cancer metastasis. J Leukoc Biol 79: 639-651, 2006.

27. Zlotnik A: Chemokines and cancer. Int J Cancer 119: 2026-2029, 2006.

28. Lu H, Ouyang W and Huang C: Inflammation, a key event in cancer development. Mol Cancer Res 4: 221-233, 2006.

29. Ben-Baruch A: The multifaceted roles of chemokines in malignancy. Cancer Metastasis Rev 25: 357-371, 2006.

30. Platten M, Kretz A, Naumann U, Aulwurm S, Egashira K, Isenmann S and Weller M: Monocyte chemoattractant protein-1 increases microglial infiltration and aggressiveness of gliomas. Ann Neurol 54: 388-392, 2003.

31. Allavena P, Sica A, Solinas G, Porta C and Mantovani A: The inflammatory micro-environment in tumor progression: the role of tumor-associated macrophages. Crit Rev Oncol Hematol 66: $1-9,2008$

32. Sica A, Schioppa T, Mantovani A and Allavena P: Tumourassociated macrophages are a distinct M2 polarised population promoting tumour progression: potential targets of anti-cancer therapy. Eur J Cancer 42: 717-727, 2006.

33. Helm O, Held-Feindt J, Grage-Griebenow E, Reiling N, Ungefroren H, Vogel I, Krüger U, Becker T, Ebsen M, Röcken C, Kabelitz D, Schäfer H and Sebens S: Tumor-associated macrophages exhibit pro- and anti-inflammatory properties by which they impact on pancreatic tumorigenesis. Int J Cancer: Jan 23 2014 (Epub ahead of print). doi: 10.1002/ijc.28736.

34. Arenberg DA, Keane MP, DiGiovine B, Kunkel SL, Strom SR, Burdick MD, Iannettoni MD and Strieter RM: Macrophage infiltration in human non-small-cell lung cancer: the role of CC chemokines. Cancer Immunol Immunother 49: 63-70, 2000.

35. Balkwill F: Cancer and the chemokine network. Nat Rev Cancer 4: 540-550, 2004.

36. Mantovani A, Allavena P, Sozzani S, Vecchi A, Locati M and Sica A: Chemokines in the recruitment and shaping of the leukocyte infiltrate of tumors. Semin Cancer Biol 14: 155-160, 2004.

37. Ueno T, Toi M, Saji H, Muta M, Bando H, Kuroi K, Koike M, Inadera $\mathrm{H}$ and Matsushima K: Significance of macrophage chemoattractant protein-1 in macrophage recruitment, angiogenesis, and survival in human breast cancer. Clin Cancer Res 6: 3282-3289, 2000.

38. Monti P, Leone BE, Marchesi F, Balzano G, Zerbi A, Scaltrini F, Pasquali C, Calori G, Pessi F, Sperti C, Di Carlo V, Allavena P and Piemonti L: The CC chemokine MCP-1/CCL2 in pancreatic cancer progression: regulation of expression and potential mechanisms of antimalignant activity. Cancer Res 63: 7451-7461, 2003. 
39. Bachleder RE, Wendt MA and Mercurio AM: Vascular endothelial growth factor promotes breast carcinoma invasion in an autocrine manner by regulating the chemokine receptor CXCR4. Cancer Res 62: 7203-7206, 2002.

40. Schioppa T, Uranchimeg B, Saccani A, Biswas SK, Doni A, Rapisarda A, Bernasconi S, Saccani S, Nebuloni M, Vago L, Mantovani A, Melillo G and Sica A: Regulation of the chemokine receptor CXCR4 by hypoxia. J Exp Med 198: 1391-1402, 2003.

41. Schutyser E, Struyf S, Proost P, Opdenakker G, Laureys G, Verhasselt B, Peperstraete L, Van de Putte I, Saccani A, Allavena P, Mantovani A and Van Damme J: Identification of biologically active chemokine isoforms from ascetic fluid and elevated levels of CCL18/pulmonary and activation-regulated chemokine in ovarian carcinoma. J Biol Chem 277: 24584-24593, 2002.

42. Sica A, Larghi P, Mancino A, Rubino L, Porta C, Totaro MG, Rimoldi M, Biswas SK, Allavena $\mathrm{P}$ and Mantovani A: Macrophage polarization in tumor progression. Semin Cancer Biol 18: 349-355, 2008

43. Biswas SK, Gangi L, Paul S, Schioppa T, Saccani A, Sironi M, Bottazzi B, Doni A, Vincenzo B, Pasqualini F, Vago L, Nebuloni M, Mantovani A and Sica A: A distinct and unique transcriptional program expressed by tumor-associated macrophages (defective NF- $\kappa \mathrm{B}$ and enhanced IRF-3/STAT1 activation). Blood 107: 2112-2122, 2006.
44. Pyonteck SM, Akkari L, Schuhmacher AJ, Bowman RL, Sevenich L, Quail DF, Olson OC, Quick ML, Huse JT, Teijeiro V, Setty M, Leslie CS, Oei Y, Pedraza A, Zhang J, Brennan CW, Sutton JC, Holland EC, Daniel D and Joyce JA: CSF-1R inhibition alters macrophage polarization and blocks glioma progression. Nat Med 19: 1264-1272, 2013.

45. Pong WW, Walker J, Wylie T, Magrini V, Luo J, Emnett RJ, Choi J, Cooper ML, Griffith M, Griffith OL, Rubin JB, Fuller GN, Piwnica-Worms D, Feng X, Hambardzumyan D, DiPersio JF, Mardis ER and Gutmann DH: F11R is a novel monocyte prognostic biomarker for malignant glioma. PLoS One: e77571, 2013.

46. Ye XZ, Xu SL, Xin YH, Yu SC, Ping YF, Chen L, Xiao HL, Wang B, Yi L, Wang QL, Jiang XF, Yang L, Zhang P, Qian C, Cui YH, Zhang X and Bian XW: Tumor-associated microglia/macrophages enhance the invasion of glioma stem-like cells via TGF- $\beta 1$ signaling pathway. J Immunol 189: 444-453, 2012. 